\title{
On transformation between international celestial and terrestrial reference systems
}

\author{
P. Bretagnon ${ }^{1}$ and V. A. Brumberg ${ }^{2}$ \\ 1 Institut de mécanique céleste et de calcul des éphémérides, 75014 Paris, France \\ 2 Institute of Applied Astronomy, 191187 St. Petersburg, Russia
}

Received 19 February 2003 / Accepted 16 May 2003

\begin{abstract}
Based on the current IAU hierarchy of the relativistic reference systems, practical formulae for the transformation between barycentric (BCRS) and geocentric (GCRS) celestial reference systems are derived. BCRS is used to refer to ICRS, International Celestial Reference System. This transformation is given in four versions, dependent on the time arguments used for BCRS (TCB or TDB) and for GCRS (TCG or TT). All quantities involved in these formulae have been tabulated with the use of the VSOP theories (IMCCE theories of motion of the major planets) ${ }^{\star}$. In particular, these formulae may be applied to account for the indirect relativistic third-body perturbations in motion of Earth's satellites and Earth's rotation problem. We propose to use the SMART theory (IMCCE theory of Earth's rotation) in constructing the Newtonian three-dimensional spatial rotation transformation between GCRS and ITRS, the International Terrestrial Reference System. This transformation is compared with two other versions involving extra angular variables currently used by IERS, the International Earth Rotation Service. It is shown that the comparison of these three forms of the same transformation may be greatly simplified by using the proposed composite rotation formula ${ }^{\star \star}$.
\end{abstract}

Key words. relativity - reference systems - time

\section{Introduction}

Recent IAU (2000) Resolution B1 (IAU 2001) on reference systems and time scales involves much more profound use of general relativity theory (GRT) in ephemeris astronomy compared to the IAU (1991) Resolution A4 (see the explanatory supplement by Soffel et al. (2003) and the discussion on implementation of this resolution in (IERS 2002). However, there still exists some confusion in using the time scales TDB and TCB, TT and TCG, and in GRT-consistent interpretation of ICRS and ITRS, the two main reference systems (RSs). The aim of this paper is to present explicitly the transformation ICRS $\leftrightarrow$ ITRS as prescribed by the IAU Resolution B1(2000).

For astronomical practice it is sufficient to have only the reference systems ICRS and ITRS with their physical realizations given by the reference frames ICRF and ITRF, respectively. In the GRT framework, ICRS represents a four-dimensional Barycentric Celestial Reference System (BCRS) whereas ITRS represents a local four-dimensional geocentric system rotating with the Earth. For the relationship between BCRS and ITRS it is necessary to introduce one more local geocentric system with the same time scale, TCG, as used for ITRS and the same directions of the spatial axes as for BCRS. Such a Geocentric Celestial Reference System (GCRS) is introduced by the IAU Resolution B1.3 (2000). GCRS represents a system kinematically nonrotating with respect to BCRS. This system rotates dynamically with respect to BCRS. The angular velocity of this rotation has a relativistic order of smallness and is caused by the motion of the geocentre around the solar system barycentre. Indeed, the fourdimensional transformation from the barycentre to the geocentre can transform any barycentric system BRS either into DGRS,

Send offprint requests to: V. A. Brumberg, e-mail: brumberg@quasar.ipa.nw.ru

* Tables 1-20 of Appendix B containing the initial terms of the VSOP-based series for the BCRS $\leftrightarrow$ GCRS transformation are only available in electronic form at the CDS via anonymous ftp to cdsarc.u-strasbg.fr (130.79.128.5) or via http://cdsweb.u-strasbg.fr/cgi-bin/qcat?J/A+A/408/387

$\star \star$ The work on ICRS $\leftrightarrow$ GCRS transformation with the use of VSOP theories was done in February-March 2002 during the stay of the second author in IMCCE. The authors hoped to complete the second part concerning GCRS $\leftrightarrow$ ITRS transformation with the use of SMART theory in September 2002 during the visit of the first author to IAA. The grave disease of Pierre Bretagnon which tragically resulted in his death on November 17, 2002, did not permit us to complete this work. The aim to improve SMART theory by taking into account the indirect relativistic third-body perturbations as indicated in the paper also remains unachieved. The second author is publishing this paper in memoriam of Pierre Bretagnon (VAB). 
a geocentric system dynamically non-rotating with respect to BRS, or into KGRS, a geocentric system kinematically nonrotating with respect to BRS. GCRS represents just such a KGRS suitable for astrometric purposes. The systems of DGRS type are more preferable for celestial mechanics enabling one to deal with the geocentric equations of motion without the terms caused by the rotation of the system. One should note also that the classical concepts of ephemeris astronomy and the system of astronomical constants are based on Newtonian mechanics with its absolute time and absolute space. In Newtonian astronomy such concepts are invariant under the transformation from the barycentre to the geocentre and vice versa. Such invariance does not take place in relativistic astronomy. To avoid possible confusion one has to go beyond the framework of ICRS and ITRS systems.

One of the possibilities (not unique, of course) is to consider several reference systems at the barycentric and geocentric levels (Brumberg et al. 1996; Brumberg 1997). At the barycentric level, along with ICRS (BCRS) one may consider ecliptical BRSC and equatorial BRSQ systems. Their main planes are chosen to coincide with planes of the ecliptic and equator fixed for J2000.0. The coordinate time scale of all these four-dimensional systems is $t=\mathrm{TCB}$. Their spatial coordinates are designated in this paper by $\boldsymbol{x}=\left(x^{i}\right), \boldsymbol{x}_{C}=\left(x_{C}^{i}\right)$ and $\boldsymbol{x}_{Q}=\left(x_{Q}^{i}\right)$, respectively. At the geocentric level each of these three system involves two geocentric systems, dynamically (D) or kinematically (K) non-rotating with respect to the corresponding barycentric system. As a result one has at the geocentric level six geocentric systems DGRS, KGRS, DGRSC, KGRSC, DGRSQ and KGRSQ. They all have (within the post-Newtonian approximation) the coordinate time scale $u=T C G$. Their spatial coordinates are designated below by $\boldsymbol{w}=\left(w^{i}\right), \boldsymbol{w}_{C}=\left(w_{C}^{i}\right)$ and $\boldsymbol{w}_{Q}=\left(w_{Q}^{i}\right)$, respectively. If necessary, the spatial coordinates for dynamically (D) or kinematically (K) non-rotating systems may be distinguished explicitly by indicating $w^{i}$ with $q=1$ for version $\mathrm{D}$ and $q=0$ for version $\mathrm{K}$. The GCRS system as defined by the IAU Resolution B1.3 (2000) corresponds in these notations to KGRS. VSOP theories of planetary motion are constructed in BRSC (Bretagnon \& Francou 1988). SMART theory of the Earth's rotation (Bretagnon et al. 1997; 1998) is to be considered in DGRSC. Since the ecliptic may be defined reasonably only at the barycentric level, GRSC (in any version) should be regarded just as another intermediate system between ICRS and ITRS. Quite similarly, since the equator is defined reasonably only at the geocentric level, BRSQ should be regarded as one more intermediary between ICRS and ITRS.

All expressions below are given in the post-Newtonian approximation within $c^{-2}$ accuracy.

\section{Transformation between BCRS and GCRS}

This section deals with the transformation between the barycentric reference system BRS $\left(t=\mathrm{TCB}, \boldsymbol{x}=x^{i}\right)$ and geocentric reference system GRS $\left(u=\right.$ TCG, $\left.w=w^{i}\right)$. The latter system is considered in both versions, KGRS $(q=0)$ and DGRS $(q=1)$ with explicit designation, if necessary, $\underset{q}{\boldsymbol{w}}$ for the corresponding spatial coordinates.

\subsection{Direct transformation $B R S \rightarrow G R S$}

Direct transformation of BRS coordinates $x^{i}$ for instant $t$ to GRS coordinates $w^{i}$ for instant $u$ reads

$u=t-c^{-2}\left[A(t)+\boldsymbol{v}_{E} \boldsymbol{r}_{E}\right]+\ldots$,

$w^{i}=r_{E}^{i}+c^{-2}\left[\frac{1}{2} \boldsymbol{v}_{E} \boldsymbol{r}_{E} v_{E}^{i}-q \varepsilon_{i j k} F^{j} r_{E}^{k}+\bar{U}_{E}\left(t, \boldsymbol{x}_{E}\right) r_{E}^{i}+\boldsymbol{a}_{E} \boldsymbol{r}_{E} r_{E}^{i}-\frac{1}{2} \boldsymbol{r}_{E}^{2} a_{E}^{i}\right]+\ldots$

with

$\dot{A}(t)=\frac{1}{2} v_{E}^{2}+\bar{U}_{E}\left(t, x_{E}\right)$,

$\bar{U}_{E}\left(t, \boldsymbol{x}_{E}\right)=\sum_{A \neq E} \frac{G M_{A}}{r_{E A}}, \quad a_{E}^{k}=-\sum_{A \neq E} \frac{G M_{A}}{r_{E A}^{3}} r_{E A}^{k}-Q_{k}$

and

$\boldsymbol{x}_{E}=\boldsymbol{x}_{E}(t), \quad \boldsymbol{v}_{E}=\dot{\boldsymbol{x}}_{E}(t), \quad \boldsymbol{r}_{E}=\boldsymbol{x}-\boldsymbol{x}_{E}, \quad \boldsymbol{r}_{E A}=\boldsymbol{x}_{E}-\boldsymbol{x}_{A}$,

$\varepsilon_{i j k}=\frac{1}{2}(i-j)(j-k)(k-i), \quad \varepsilon_{i j k} F^{j} r_{E}^{k}=\left(\boldsymbol{F} \times \boldsymbol{r}_{E}\right)^{i}$.

Within $c^{-2}$ accuracy $\bar{U}_{E}\left(t, x_{E}\right)$ represents just the Newtonian potential (in the point-mass approximation) of all solar system bodies (except the Earth) evaluated at the geocentre (index $A$ in (4) runs over the Sun, the Moon and major planets). The Einstein summation rule (from 1 to 3 ) is applied everywhere for any twice repeated Latin index unless indicated otherwise. Functions $Q_{k}$ in the acceleration of the Earth $a_{E}^{k}$ represent small contributions caused by the non-geodesic BRS motion of the Earth due to the interaction of its quadrupole moments $\left(I_{E}^{m n}\right)$ and the external masses

$Q_{k}=-\frac{1}{2} M_{E}^{-1} I_{E}^{m n} \bar{U}_{E, k m n}\left(t, \boldsymbol{x}_{E}\right)$

with the comma designating here and below a partial derivative with respect to the corresponding coordinate $x^{i}$ before the substitution $x^{i}=x_{E}^{i}$. 


\subsection{Inverse transformation $G R S \rightarrow B R S$}

The inverse transformation to transform GRS coordinates $w^{i}$ for instant $u$ to BRS coordinates $x^{i}$ for instant $t$ reads

$t=u+c^{-2}\left[A(u)+\boldsymbol{v}_{E} \boldsymbol{w}\right]+\ldots$,

$x^{i}=w^{i}+z_{E}^{i}(u)+c^{-2}\left[\frac{1}{2} \boldsymbol{v}_{E} \boldsymbol{w} v_{E}^{i}+q \varepsilon_{i j k} F^{j} w^{k}-\bar{U}_{E}\left(t, \boldsymbol{x}_{E}\right) w^{i}-\boldsymbol{a}_{E} \boldsymbol{w} w^{i}+\frac{1}{2} \boldsymbol{w}^{2} a_{E}^{i}\right]+\ldots$

with the relativistic time equation

$u=t^{*}-c^{-2} A\left(t^{*}\right)+\ldots$,

and the TCG representation of the motion of the Earth

$z_{E}^{i}(u)=x_{E}^{i}\left(t^{*}\right)$

For direct transformations (1) and (2) one should know the function $A=A(t)$ by integrating (3) numerically or analytically. For the inverse transformation (7) and (8) to express the right-hand members in terms of $u$ one should get function $t^{*}=t^{*}(u)$ by inverting the equation (9) also in numerical or analytical form. Some analytical expressions for it will be given below. The Eq. (9) results from the transformation (7) applied in the geocentre.

\subsection{Geodesic rotation}

The geodesic rotation vector $F^{i}$ including geodetic precession, geodetic nutation and luni-solar-planetary terms (under the nonrotating point-mass model) is computed by integrating the expression

$\dot{F}^{i}=\sum_{A \neq E} \frac{G M_{A}}{r_{E A}^{3}}\left[\left(-\frac{3}{2} \boldsymbol{v}_{E}+2 \boldsymbol{v}_{A}\right) \times \boldsymbol{r}_{E A}\right]^{i}$.

Indeed, the complete expression for $\dot{F}^{i}$ reads (e.g., Brumberg 1991)

$\dot{F}^{i}=\varepsilon_{i j k}\left[\frac{3}{2} v_{E}^{j} a_{E}^{k}-2 \bar{U}_{E, k}^{j}\left(t, \boldsymbol{x}_{E}\right)+2 v_{E}^{j} Q_{k}\right]$

or else

$\dot{F}^{i}=\varepsilon_{i j k}\left[\frac{3}{2} v_{E}^{j} \bar{U}_{E, k}\left(t, \boldsymbol{x}_{E}\right)-2 \bar{U}_{E, k}^{j}\left(t, \boldsymbol{x}_{E}\right)+\frac{1}{2} v_{E}^{j} Q_{k}\right]$

where $\bar{U}_{E}^{j}\left(t, \boldsymbol{x}_{E}\right)$ stands for the external (with respect to the Earth) vector-potential evaluated at the geocentre. In neglecting in $\bar{U}_{E}^{j}$ all contributions from the non-point structure and proper rotations of the bodies one has

$\bar{U}_{E}^{j}\left(t, \boldsymbol{x}_{E}\right)=\sum_{A \neq E} \frac{G M_{A}}{r_{E A}} v_{A}^{j}, \quad \bar{U}_{E, k}^{j}\left(t, \boldsymbol{x}_{E}\right)=-\sum_{A \neq E} \frac{G M_{A}}{r_{E A}^{3}} r_{E A}^{k} v_{A}^{j}$.

In combination with (4) it results in (11) if one neglects also the terms with $Q_{k}$. It presents no difficulties to take into account the figure and spin dependent terms in the vector-potential $\bar{U}_{E}^{j}\left(t, \boldsymbol{x}_{E}\right)$. Some estimates show (Vokrouhlický 1995) that solar spin terms may be significant. However, due to possible uncertainty of such terms we prefer to use in (11) the model of point masses. Explicit expressions for $\dot{F}_{C}^{i}$ and $F_{C}^{i}$ (geodesic rotation in BRSC) are given in Brumberg \& Bretagnon (2001). For BRSC (ecliptical $\mathrm{BRS}$ ) and BRSQ (equatorial BRS) one has

$\boldsymbol{x}_{C}=P_{C} \boldsymbol{x}, \quad \boldsymbol{x}_{Q}=P_{Q} \boldsymbol{x}$

and matrix relations

$F=P_{C}^{\mathrm{T}} F_{C} P_{C}, \quad F=P_{Q}^{\mathrm{T}} F_{Q} P_{Q}$

with the relationships between matrix elements $F^{i j}$ and vector components $F^{i}$ as follows:

$F^{i}=\frac{1}{2} \varepsilon_{i j k} F^{j k}, \quad F^{i j}=\varepsilon_{i j k} F^{k}$. 


\subsection{Constant rotations}

By comparing IMCCE analytical VSOP theories constructed in BRSC with JPL numerical ephemerides DE-405 constructed in ICRS one can estimate the constant rotation matrices $P_{C}$ and $P_{Q}$ (Bretagnon 2002; Bretagnon et al. 2003)

$P_{C}=D_{1}(\varepsilon) D_{3}(\chi), \quad P_{Q}=D_{3}(\chi)$

$\varepsilon=23^{\circ} 26^{\prime} 21.408800^{\prime \prime}=0.409092614174, \quad \chi=-0.053727^{\prime \prime}=-0.000000260476$,

$D_{i}(\alpha)$ being elementary rotation matrices

$D_{1}(\alpha)=\left(\begin{array}{ccc}1 & 0 & 0 \\ 0 & \cos \alpha & \sin \alpha \\ 0 & -\sin \alpha & \cos \alpha\end{array}\right), D_{2}(\alpha)=\left(\begin{array}{ccc}\cos \alpha & 0 & -\sin \alpha \\ 0 & 1 & 0 \\ \sin \alpha & 0 & \cos \alpha\end{array}\right), D_{3}(\alpha)=\left(\begin{array}{ccc}\cos \alpha & \sin \alpha & 0 \\ -\sin \alpha & \cos \alpha & 0 \\ 0 & 0 & 1\end{array}\right)$.

\subsection{Constants of integration}

Both the time-function $A(t)$ and geodetic rotation vector $F^{i}(t)$ are defined only by means of their time derivatives (3) and (11), respectively, involving additive arbitrary constants to be determined from complementary considerations. The necessity to introduce such a constant in $A(t)$ was discussed in detail by Guinot (2000). Guinot suggested to determine this constant so that $t=u$ on January 1, 1977 0h 0m 0s TAI (JD = 2443144.5 TAI) at the geocentre (1977 Origin) as prescribed by IAU Resolution A4 (1991). In what follows, the function $A(t)$ is always considered taking into account this condition.

In the same manner, one may define a constant value in $F^{i}$. But in this case it is reasonable to take $\mathrm{J} 2000.0$ as the fundamental epoch recommended by IAU (1994) Resolution C7 (the epoch defined at the geocentre at the date January $1.5,2000$ TT = JD 2451545.0 TT). Indeed, J2000.0 is the fundamental epoch for astronomical reference systems and it is logically to demand $F^{i}=0$ at this epoch. This value does not affect the relationship between BCRS and GCRS but it is of importance for the definition of DGRSs, i.e. dynamically non-rotating geocentric systems. The geodetic rotation vector $F^{i}$ determines the mutual rotation of DGRS and KGRS but under the proposed choice of the constant of integration, these systems coincide at the epoch J2000.0 (the values of $F_{C}^{i}$ in Brumberg \& Bretagnon 2001 are given without such a constant term).

\subsection{Time scales}

The currently employed time scales satisfy the relations (see, for example, BDL 1997):

$\mathrm{TT}=\mathrm{TAI}+32.184 \mathrm{~s}, \quad \mathrm{TDB}=\mathrm{TT}+P+c^{-2} \boldsymbol{v}_{E} \boldsymbol{w}$,

$\mathrm{TCG}-\mathrm{TT}=L_{G}(J-2443144.5) 86400 \mathrm{~s}, \quad L_{G}=6.969290134 \times 10^{-10}$,

$\mathrm{TCB}-\mathrm{TDB}=L_{B}(J-2443144.5) 86400 \mathrm{~s}$,

$\mathrm{TCB}-\mathrm{TCG}=L_{C}(J-2443144.5) 86400 \mathrm{~s}+P+c^{-2} \boldsymbol{v}_{E} \boldsymbol{w}$,

$L_{C}=1.48082686741 \times 10^{-8}, \quad L_{B}=1.55051976772 \times 10^{-8}$.

$L_{B}, L_{C}, L_{G}$ values are taken here from IAU (2001). $L_{G}$ is now fixed by the IAU Resolution B1 (2000) as a defining constant. By contrast, $L_{C}$ and hence $L_{B}\left(=L_{C}+L_{G}-L_{C} L_{G}\right)$ depend on specific planetary and lunar theories employed in integrating (3) and are different for different sets of planetary and lunar ephemerides. In the next section we give data based on using VSOP ephemerides involving $L_{C}$ and $L_{B}$ values slightly different from (22). $P$ in (18) and (21) denotes the quasi-trigonometric part of function $A(t)$ vanishing at the 1977 Origin $\left(A(t)\right.$ without its linear secular trend $\left.c^{2} L_{C} t\right)$. As is well known, TAI is the only time scale determined in practice from averaging the readings of the clocks synchronized with respect to TT. In other words, TAI represents the physical realization of TT.

The function $A(t)$ satisfying (3) is presented usually (Fairhead \& Bretagnon 1990; BDL 1997) as

$A(t)=c^{2} L_{C} t+A_{p}(t)$

with

$L_{C} t=L_{C}(J-2443144.5) 86400 s$

and $A_{p}(t)=P$ determined either numerically or in the analytical form

$A_{p}(t)=\sum_{\alpha} t^{\alpha}\left[\sum_{k} A_{k}^{\alpha} \cos \left(\psi_{k}^{\alpha}+v_{k}^{\alpha} t\right)\right]$. 
It is to be noted that most existing solutions for this function have been obtained so far in terms of TDB with no additional constant discussed above (see Fukushima 1995; Irwin \& Fukushima 1999 and references therein).

The representation (23) enables one to transform (9) into

$u=\left(1-L_{C}\right) t^{*}-c^{-2} A_{p}\left(t^{*}\right)+\ldots$

Therefore, the inversion of (9) may be written either as

$t^{*}=u+c^{-2} A(u)$

or else

$t^{*}=\left(1+L_{C}\right) u+c^{-2} A_{p}(u)$.

These two expressions result in the function (10) in the form

$z_{E}^{i}(u)=x_{E}^{i}(u)+c^{-2} A(u) v_{E}^{i}(u)+\ldots$

or

$z_{E}^{i}(u)=x_{E}^{i}\left[\left(1+L_{C}\right) u\right]+c^{-2} A_{p}(u) v_{E}^{i}(u)+\ldots$

(within the post-Newtonian accuracy one may neglect the relativistic differences in the arguments of the relativistic terms). Considering that the functions $x_{E}^{i}(t)$ have in the present analytical theories the form

$x_{E}^{i}(t)=\sum_{\alpha} t^{\alpha}\left[\sum_{k} X_{i k}^{\alpha} \cos \left(\psi_{k}^{\alpha}+v_{k}^{\alpha} t\right)\right]$.

The presentation (30) is more advantageous retaining the $L_{C}$ factor within the arguments of the leading trigonometric terms. Note again that the analytical ephemerides obtained in BRSC should be first converted into BCRS in accordance with (15), i.e.

$\boldsymbol{x}=P_{C}^{-1} \boldsymbol{x}_{C}=D_{3}(-\chi) D_{1}(-\varepsilon) \boldsymbol{x}_{C}$.

Hence, returning to the standard designations for the time-scales one has

$\mathrm{TCB}^{*}=\left(1+L_{C}\right) \mathrm{TCG}+c^{-2} A_{p} \quad(t=\mathrm{TCB}, u=\mathrm{TCG})$.

Instead of TCB and TCG one often makes use of TDB and TT, respectively, involving the scaling transformations (Brumberg \& Groten 2001a)

$\mathrm{TDB}=\left(1-L_{B}\right) \mathrm{TCB}, \quad(\boldsymbol{x})_{\mathrm{TDB}}=\left(1-L_{B}\right) \boldsymbol{x}, \quad(G M)_{\mathrm{TDB}}=\left(1-L_{B}\right) G M$

and

$\mathrm{TT}=\left(1-L_{G}\right) \mathrm{TCG}, \quad(\boldsymbol{w})_{\mathrm{TT}}=\left(1-L_{G}\right) \boldsymbol{w}, \quad(G \hat{M})_{\mathrm{TT}}=\left(1-L_{G}\right)(G \hat{M})$.

Therefore, along with (33) one has three more expressions depending on the employed arguments

$\mathrm{TDB}^{*}=\left(1-L_{G}\right) \mathrm{TCG}+c^{-2} A_{p} \quad(t=\mathrm{TDB}, u=\mathrm{TCG})$,

$\mathrm{TCB}^{*}=\left(1+L_{B}\right) \mathrm{TT}+c^{-2} A_{p} \quad(t=\mathrm{TCB}, u=\mathrm{TT})$,

$\mathrm{TDB}^{*}=\mathrm{TT}+c^{-2} A_{p} \quad(t=\mathrm{TDB}, u=\mathrm{TT})$.

The asterisk in (33), (36)-(38) has the same meaning as in (9) relating the BRS event $\left(t^{*}, \boldsymbol{x}=\boldsymbol{x}_{E}\right)$ and the GRS event $(u, \boldsymbol{w}=0)$. 


\subsection{Working transformation formulae}

To summarize, one has for the BRS $\leftrightarrow$ GRS transformation four versions as follows:

1. BRS with TCB, GRS with TCG:

Direct transformation:

$\mathrm{TCG}=\left(1-L_{C}\right) \mathrm{TCB}-c^{-2}\left(A_{p}+\boldsymbol{v}_{E} \boldsymbol{r}_{E}\right)$,

$w^{i}=r_{E}^{i}+c^{-2} \Lambda^{i}\left(t, \boldsymbol{r}_{E}\right)+\ldots$

with

$\Lambda^{i}\left(t, \boldsymbol{r}_{E}\right)=\frac{1}{2} \boldsymbol{v}_{E} \boldsymbol{r}_{E} v_{E}^{i}-q \varepsilon_{i j k} F^{j} r_{E}^{k}+\bar{U}_{E}\left(t, \boldsymbol{x}_{E}\right) r_{E}^{i}+\boldsymbol{a}_{E} \boldsymbol{r}_{E} r_{E}^{i}-\frac{1}{2} \boldsymbol{r}_{E}^{2} a_{E}^{i}$.

Inverse transformation:

$\mathrm{TCB}=\left(1+L_{C}\right) \mathrm{TCG}+c^{-2}\left(A_{p}+\boldsymbol{v}_{E} \boldsymbol{w}\right)$,

$x^{i}=w^{i}+z_{E}^{i}(\mathrm{TCG})+c^{-2} \Gamma^{i}(u, w)+\ldots$

with

$z_{E}^{i}(\mathrm{TCG})=x_{E}^{i}\left(\mathrm{TCB}^{*}\right)=x_{E}^{i}\left[\left(1+L_{C}\right) \mathrm{TCG}\right]+c^{-2} A_{p} v_{E}^{i}+\ldots$

and

$\Gamma^{i}(u, \boldsymbol{w})=\frac{1}{2} \boldsymbol{v}_{E} \boldsymbol{w} v_{E}^{i}+q \varepsilon_{i j k} F^{j} w^{k}-\bar{U}_{E}\left(t, \boldsymbol{x}_{E}\right) w^{i}-\boldsymbol{a}_{E} \boldsymbol{w} w^{i}+\frac{1}{2} \boldsymbol{w}^{2} a_{E}^{i}$.

Since $\Lambda^{i}\left(t, \boldsymbol{r}_{E}\right)$ and $\Gamma^{i}(u, w)$ represent post-Newtonian corrections there is no need to distinguish here between $t, \boldsymbol{r}_{E}$ on the one hand and $u, \boldsymbol{w}$ on the other hand. Evidently, $\Lambda^{i}\left(t, \boldsymbol{r}_{E}\right)+\Gamma^{i}(u, \boldsymbol{w})=\boldsymbol{v}_{E} \boldsymbol{w} v_{E}^{i}$.

2. BRS with TDB, GRS with TCG:

Direct transformation:

$\mathrm{TCG}=\left(1+L_{G}\right) \mathrm{TDB}-c^{-2}\left(A_{p}+\boldsymbol{v}_{E} \boldsymbol{r}_{E}\right)$,

$w^{i}=\left(1+L_{B}\right)\left(r_{E}^{i}\right)_{\mathrm{TDB}}+c^{-2} \Lambda^{i}\left(t, \boldsymbol{r}_{E}\right)+\ldots$

Inverse transformation:

$\mathrm{TDB}=\left(1-L_{G}\right) \mathrm{TCG}+c^{-2}\left(A_{p}+\boldsymbol{v}_{E} \boldsymbol{w}\right)$,

$\left(x^{i}\right)_{\mathrm{TDB}}=\left(1-L_{B}\right) w^{i}+\left(1-L_{B}\right) z_{E}^{i}(\mathrm{TCG})+c^{-2} \Gamma^{i}(u, w)+\ldots$

with

$\left(1-L_{B}\right) z_{E}^{i}(\mathrm{TCG})=x_{E}^{i}\left(\mathrm{TDB}^{*}\right)=x_{E}^{i}\left[\left(1-L_{G}\right) \mathrm{TCG}\right]+c^{-2} A_{p} v_{E}^{i}+\ldots$

3. BRS with TCB, GRS with TT:

Direct transformation:

$\mathrm{TT}=\left(1-L_{B}\right) \mathrm{TCB}-c^{-2}\left(A_{p}+\boldsymbol{v}_{E} \boldsymbol{r}_{E}\right)$,

$\left(w^{i}\right)_{\mathrm{TT}}=\left(1-L_{G}\right) r_{E}^{i}+c^{-2} \Lambda^{i}\left(t, \boldsymbol{r}_{E}\right)+\ldots$

Inverse transformation:

$\mathrm{TCB}=\left(1+L_{B}\right) \mathrm{TT}+c^{-2}\left(A_{p}+\boldsymbol{v}_{E} \boldsymbol{w}\right)$,

$x^{i}=\left(1+L_{G}\right)\left(w^{i}\right)_{\mathrm{TT}}+\left(1+L_{G}\right) z_{E}^{i}(\mathrm{TT})+c^{-2} \Gamma^{i}(u, \boldsymbol{w})+\ldots$

with

$\left(1+L_{G}\right) z_{E}^{i}(\mathrm{TT})=x_{E}^{i}\left(\mathrm{TCB}^{*}\right)=x_{E}^{i}\left[\left(1+L_{B}\right) \mathrm{TT}\right]+c^{-2} A_{p} v_{E}^{i}+\ldots$ 
4. BRS with TDB, GRS with TT:

Direct transformation:

$\mathrm{TT}=\mathrm{TDB}-c^{-2}\left(A_{p}+\boldsymbol{v}_{E} \boldsymbol{r}_{E}\right)$,

$\left(w^{i}\right)_{\mathrm{TT}}=\left(1+L_{C}\right)\left(r_{E}^{i}\right)_{\mathrm{TDB}}+c^{-2} \Lambda^{i}\left(t, \boldsymbol{r}_{E}\right)+\ldots$

Inverse transformation:

$\mathrm{TDB}=\mathrm{TT}+c^{-2}\left(A_{p}+\boldsymbol{v}_{E} \boldsymbol{w}\right)$

$\left(x^{i}\right)_{\mathrm{TDB}}=\left(1-L_{C}\right)\left(w^{i}\right)_{\mathrm{TT}}+\left(1-L_{C}\right) z_{E}^{i}(\mathrm{TT})+c^{-2} \Gamma^{i}(u, w)+\ldots$

with

$\left(1-L_{C}\right) z_{E}^{i}(\mathrm{TT})=x_{E}^{i}\left(\mathrm{TDB}^{*}\right)=x_{E}^{i}(\mathrm{TT})+c^{-2} A_{p} v_{E}^{i}+\ldots$

In all these expressions $\Lambda^{i}\left(t, \boldsymbol{r}_{E}\right)$ and $\Gamma^{i}(u, w)$ are determined by (41) and (45), respectively. Note that for BCRS $\leftrightarrow$ GCRS transformation one should always put $q=0$.

\subsection{Geocentric third-body position vectors}

It is to be noted that the function $z_{E}^{i}(u)$ determined by one of four options (44), (50), (55), (60) as well as the similar function $z_{A}^{i}(u)=x_{A}^{i}\left(t^{*}\right)$ determined in the same manner for any body $A$ are of importance in computing indirect relativistic perturbations in the geocentric motion of Earth's satellites, Earth's rotation problem (precession-nutation theory), etc. In such problems, in neglecting direct relativistic perturbations one has to deal with Newtonian equations of motion in DGRS where the right-hand members are functions of $u$ and geocentric position vectors $w_{A}(u)$ for perturbing bodies $A$. The coordinates of the perturbing bodies are known in BRS in terms of time $t$. Denoting

$\boldsymbol{r}_{A E}=\boldsymbol{x}_{A}-\boldsymbol{x}_{E}, \quad \boldsymbol{v}_{A E}=\boldsymbol{v}_{A}-\boldsymbol{v}_{E}$

and using functions $z_{E}^{i}(u), z_{A}^{i}(u)$ one may express the geocentric position vectors of the disturbing bodies as follows (Brumberg 1996):

$w_{A}^{i}(u)=z_{A}^{i}(u)-z_{E}^{i}(u)+c^{-2}\left[\Lambda^{i}\left(t^{*}, \boldsymbol{r}_{A E}\right)+\boldsymbol{v}_{E} \boldsymbol{r}_{A E} v_{A E}^{i}\right]$

where $\Lambda^{i}\left(t^{*}, \boldsymbol{r}_{A E}\right)$ is determined by (41) replacing $\boldsymbol{r}_{E}$ by $\boldsymbol{r}_{A E}$. This transformation enables one to take into account in DGRS the indirect relativistic perturbations and to transform BRS arguments into GRS arguments.

\section{Transformation between BCRS and GCRS with VSOP data}

We have used the VSOP theories to compute the initial terms of the series for $\boldsymbol{x}_{E}, \boldsymbol{v}_{E}, c^{-2} \boldsymbol{a}_{E}, c^{-2} \bar{U}_{E}\left(t, \boldsymbol{x}_{E}\right), c^{-2} A_{p}, c^{-2} A_{p} \boldsymbol{v}_{E}$, and $c^{-2} \boldsymbol{F}$ in function of $t=$ TDB. These series are to be used to evaluate explicitly the coefficients in (56)-(60). For the sake of completeness we computed also the series for $c^{-2} \dot{\boldsymbol{F}}$ needed when considering the equations of motion in GCRS. All values have been computed using the astronomical unit as the unit of length and 1000 Julian years (365250 Julian days) as the unit of time. The value of $L_{C}$ obtained with the VSOP solution reads

$L_{C}=1.480826855667 \times 10^{-8}$.

In integrating the equations for $\dot{A}$ and $\dot{\boldsymbol{F}}$ the additive constants were introduced to annul these quantities for the 1977 Origin and $\mathbf{J} 2000.0$, respectively.

All series are presented in the form of (31) in Tables 1-20 containing the ordinal number of the term, components of the trigonometric argument (mean longitudes of eight major planets from Mercury to Neptune and arguments $D, F, l$ of the lunar theory as described in (Bretagnon et al. 1988) given to show the physical meaning of the term, coefficient $X$, the phase angle $\psi$ of the argument, the frequency $v$ of the argument and exposant $\alpha$ of power of $t$.

These series are available as Appendix B to the present paper. 


\section{Transformation between ITRS and GCRS}

If $\underset{0}{\hat{P}}(u)$ denotes the Earth's rotation matrix relating ITRS and KGRSC (geocentric kinematically non-rotating ecliptical reference system) and $\boldsymbol{y}$ is the triplet of the spatial coordinates of ITRS then ITRS $\leftrightarrow$ GCRS transformation has the form (Brumberg $\&$ Groten 2001b)

$\boldsymbol{y}=T \underset{0}{w}$

with

$T=\underset{0}{\hat{P}}(u) P_{C}$.

The rotation matrix $\underset{0}{\hat{P}}(u)$ is defined by its three Euler angles. This representation brings (64) to the form

$T=\underset{0}{D_{3}}(\underset{0}{\varphi}) D_{1}(-\underset{0}{\theta}) D_{3}(-\underset{0}{\psi}) D_{1}(\varepsilon) D_{3}(\chi)$

It is to be noted that the SMART theory involves dynamical Euler angles of matrix $\hat{P}(u)$ relating ITRS and DGRSC (geocentric dynamically non-rotating ecliptical reference system) but the transformation between dynamical and kinematical Euler angles presents no difficulties (Brumberg 1997; Bretagnon et al. 1997).

On the other hand, along with this "dynamical" expression coming from the dynamical treatment of the Earth's rotation problem there is a "kinematical" expression (e.g., Moritz \& Mueller 1987 and different modifications in IERS documents)

$T=D_{2}\left(-x_{p}\right) D_{1}\left(-y_{p}\right) D_{3}(\mathrm{GAST}) N P$

$N=D_{1}(-\varepsilon-\Delta \varepsilon) D_{3}(-\Delta \psi) D_{1}(\varepsilon)$

$P=D_{3}\left(-z_{A}\right) D_{2}\left(\theta_{A}\right) D_{3}\left(-\zeta_{A}\right)$

used in astrometry and IERS programs. Here $x_{p}$ and $y_{p}$ are the polar motion parameters, GAST stands for the Greenwich Apparent Sidereal Time, $\zeta_{A}, z_{A}$ and $\theta_{A}$ are the equatorial precession parameters and $\Delta \psi, \Delta \varepsilon$ are the astronomic nutation components in longitude and in obliquity, respectively. Recently, IAU has recommended to use the representation of rotation $T$ based on the notion of "non-rotating origin" (Capitaine et al. 2000; IERS 2003)

$T=D_{2}\left(-x_{p}\right) D_{1}\left(-y_{p}\right) D_{3}\left(s^{\prime}\right) D_{3}(\theta) D_{3}(-s) D_{3}(-E) D_{2}(d) D_{3}(E)$

under standard designations of the indicated papers.

The relationships between the representations (65), (66) and (69) of the Earth's rotation may be analysed by using the technique of Folkner et al. (1994). This technique is based on the rotation formula

$T=R(\boldsymbol{A}), \quad R(\boldsymbol{A}) \boldsymbol{x}=\boldsymbol{x}-\sin a(\hat{\boldsymbol{A}} \times \boldsymbol{x})+(1-\cos a)[\hat{\boldsymbol{A}} \times(\hat{\boldsymbol{A}} \times \boldsymbol{x})]$

where $a=|\boldsymbol{A}|$ is the rotation angle, $\hat{\boldsymbol{A}}=\boldsymbol{A} / a$ is the unit vector along the rotation axis, $\boldsymbol{x}$ is an arbitrary coordinate vector, and $T=R(\boldsymbol{A})$ is the matrix of rotation about the arbitrary axis $\hat{\boldsymbol{A}}$. With this notation the elementary rotations (17) will be

$D_{k}(\alpha)=R\left(\alpha \boldsymbol{e}_{k}\right)$

$\boldsymbol{e}_{k}$ being a unit vector along $x^{k}$ axis. The technique of Folkner et al. (1994) makes use of two relations

$M R(N) M^{T}=R(M N)$,

valid for any rotation matrix $M$, and

$R(\boldsymbol{B}) R(\boldsymbol{A}) \approx R\left[\boldsymbol{A}+\boldsymbol{B}+\frac{1}{2}(\boldsymbol{A} \times \boldsymbol{B})\right]$,

valid for small rotation vectors $\boldsymbol{A}$ and $\boldsymbol{B}$ through second order. Expressions (70)-(73) result from the theory of rotations of analytical dynamics (e.g., Pars 1965) but to simplify discussion we give their straightforward derivation in Appendix A. In addition to (73) we will need also the first-order composite rotation formula $R(\boldsymbol{B}) R(\boldsymbol{A})$ under the assumption that only one of two vectors $\boldsymbol{A}$ or $\boldsymbol{B}$ is small. Assuming that vector $\boldsymbol{B}$ is small these composite first-order (with respect to $\boldsymbol{B}$ ) formulae read

$R(\boldsymbol{B}) R(\boldsymbol{A})=R\left[\boldsymbol{A}+\frac{a(1+\cos a)}{2 \sin a} \boldsymbol{B}+\frac{1}{2}(\boldsymbol{A} \times \boldsymbol{B})+\left(1-\frac{a(1+\cos a)}{2 \sin a}\right) \hat{\boldsymbol{A}}(\hat{\boldsymbol{A}} \boldsymbol{B})\right]$ 
and

$R(\boldsymbol{A}) R(\boldsymbol{B})=R\left[\boldsymbol{A}+\frac{a(1+\cos a)}{2 \sin a} \boldsymbol{B}-\frac{1}{2}(\boldsymbol{A} \times \boldsymbol{B})+\left(1-\frac{a(1+\cos a)}{2 \sin a}\right) \hat{\boldsymbol{A}}(\hat{\boldsymbol{A}} \boldsymbol{B})\right]$.

These formulae are derived in Appendix A. Combination of (74) and (75) results in the first-order composition formula for small rotation vectors $\boldsymbol{B}$ and $\boldsymbol{C}$

$R(\boldsymbol{C}) R(\boldsymbol{A}) R(\boldsymbol{B})=R\left[\boldsymbol{A}+\frac{a(1+\cos a)}{2 \sin a}(\boldsymbol{B}+\boldsymbol{C})-\frac{1}{2}(\boldsymbol{A} \times(\boldsymbol{B}-\boldsymbol{C}))+\left(1-\frac{a(1+\cos a)}{2 \sin a}\right) \hat{\boldsymbol{A}}(\hat{\boldsymbol{A}}(\boldsymbol{B}+\boldsymbol{C}))\right]$.

Let us note also that for small $\boldsymbol{A}$ both formulae (74) and (75) result in the simple second-order formula (73). In representation (65) the three Euler angles $\varphi_{0}, \theta$ and $\psi$ can be determined on the basis of the Earth's rotation theory such as SMART97 (Bretagnon et al. 1997, 1998). Such theory involves the representation

$\underset{0}{\theta}=\boldsymbol{\varepsilon}+\underset{0}{\theta}$

From the defining expression for $D_{1}$ we get the first-order relation

$D_{1}(-\varepsilon-\underset{0}{\delta \theta})=D_{1}(-\delta \underset{0}{\theta}) D_{1}(-\varepsilon)$.

On the other hand, by applying (72) one gets

$D_{1}(-\varepsilon) D_{3}\left(-\underset{0}{\psi} D_{1}(\varepsilon)=R\left(\begin{array}{c}0 \\ \psi \sin \varepsilon \\ 0 \\ -\underset{0}{\psi} \cos \varepsilon\end{array}\right)\right.$.

Therefore, the transformation matrix (65) can be represented as follows:

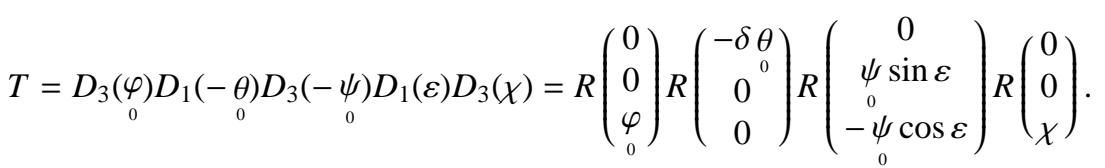

Considering that the angles $\underset{0}{\psi}, \delta \underset{0}{\theta}$ and $\chi$ are small one gets with the aid of (73) the first-order formula

$T=R\left(\begin{array}{l}0 \\ 0 \\ \varphi \\ 0\end{array}\right) R\left(\begin{array}{c}-\delta \theta \\ 0 \\ \psi \sin \varepsilon \\ 0 \\ -\psi \cos \varepsilon+\chi \\ 0\end{array}\right)$

It reduces to the composite rotation formula (75) with

$\boldsymbol{A}=\left(\begin{array}{l}0 \\ 0 \\ \varphi \\ 0\end{array}\right), \quad \boldsymbol{B}=\left(\begin{array}{c}-\delta \theta \\ 0 \\ \psi \sin \varepsilon \\ 0 \\ -\psi \cos \varepsilon+\chi \\ 0\end{array}\right)$

Now let us return to the classical astrometric transformation, i.e. (66)-(68). Using again (72) and (73) one may transform it to the product of four rotation vectors, i.e.

$D_{2}\left(-x_{p}\right) D_{1}\left(-y_{p}\right) D_{3}(\mathrm{GAST})=R\left(\begin{array}{c}-y_{p} \\ -x_{p} \\ 0\end{array}\right) R\left(\begin{array}{c}0 \\ 0 \\ \varphi_{G}\end{array}\right), \quad \varphi_{G}=\mathrm{GAST}$,

$N=R\left(\begin{array}{c}-\Delta \varepsilon \\ \Delta \psi \sin \varepsilon \\ -\Delta \psi \cos \varepsilon\end{array}\right)$

$P=R\left(\begin{array}{l}p^{1} \\ p^{2} \\ p^{3}\end{array}\right)$, 
$p^{i}$ being components of the vector of precession. Multiplication of these vectors by using (72) results to the first-order formula $T=R\left(\begin{array}{c}-y_{p} \\ -x_{p} \\ 0\end{array}\right) R\left(\begin{array}{c}0 \\ 0 \\ \varphi_{G}\end{array}\right) R\left(\begin{array}{c}-\Delta \varepsilon+p^{1} \\ \sin \varepsilon \Delta \psi+p^{2} \\ -\cos \varepsilon \Delta \psi+p^{3}\end{array}\right)$

It reduces to the composite rotation formula (76) with

$\boldsymbol{A}=\left(\begin{array}{c}0 \\ 0 \\ \varphi_{G}\end{array}\right), \quad \boldsymbol{B}=\left(\begin{array}{c}-\Delta \varepsilon+p^{1} \\ \sin \varepsilon \Delta \psi+p^{2} \\ -\cos \varepsilon \Delta \psi+p^{3}\end{array}\right), \quad \boldsymbol{C}=\left(\begin{array}{c}-y_{p} \\ -x_{p} \\ 0\end{array}\right)$

In using the modern astrometric transformation (69) by virtue of

$D_{2}(d)=R\left(d e_{2}\right), \quad D_{3}\left(s^{\prime}\right) D_{3}(\theta) D_{3}(-s)=D_{3}\left(\theta-s+s^{\prime}\right)$

and

$D_{3}(-E) D_{2}(d) D_{3}(E)=R\left(\begin{array}{c}-d \sin E \\ d \cos E \\ 0\end{array}\right)$

one has

$T=R\left(\begin{array}{c}-y_{p} \\ -x_{p} \\ 0\end{array}\right) R\left(\begin{array}{c}0 \\ 0 \\ \theta-s+s^{\prime}\end{array}\right) R\left(\begin{array}{c}-d \sin E \\ d \cos E \\ 0\end{array}\right)$

It reduces to (76) with

$\boldsymbol{A}=\left(\begin{array}{c}0 \\ 0 \\ \theta-s+s^{\prime}\end{array}\right), \quad \boldsymbol{B}=\left(\begin{array}{c}-d \sin E \\ d \cos E \\ 0\end{array}\right), \quad \boldsymbol{C}=\left(\begin{array}{c}-y_{p} \\ -x_{p} \\ 0\end{array}\right)$

Finally, for vector $\boldsymbol{A}$ in representation (70) of transformation (63) one has three first-order expressions as follows:

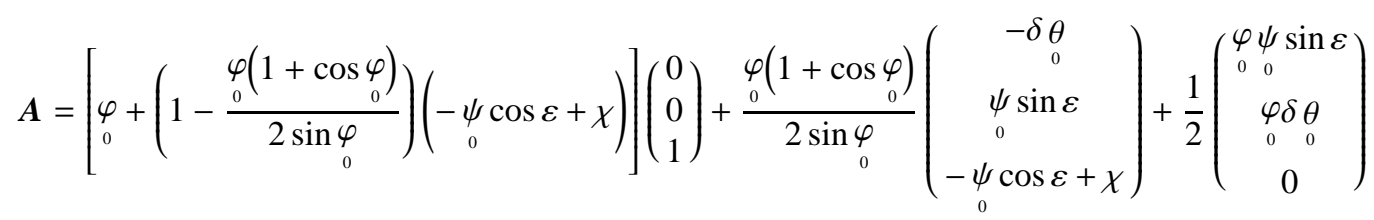

in the case of (4.3),

$$
\begin{aligned}
\boldsymbol{A}= & {\left[\varphi_{G}+\left(1-\frac{\varphi_{G}\left(1+\cos \varphi_{G}\right)}{2 \sin \varphi_{G}}\right)\left(-\Delta \psi \cos \varepsilon+p^{3}\right)\right]\left(\begin{array}{l}
0 \\
0 \\
1
\end{array}\right) } \\
& +\frac{\varphi_{G}\left(1+\cos \varphi_{G}\right)}{2 \sin \varphi_{G}}\left(\begin{array}{c}
-\Delta \varepsilon+p^{1}-y_{p} \\
\Delta \psi \sin \varepsilon+p^{2}-x_{p} \\
-\Delta \psi \cos \varepsilon+p^{3}
\end{array}\right)+\frac{1}{2}\left(\begin{array}{c}
\varphi_{G}\left(\Delta \psi \sin \varepsilon+p^{2}+x_{p}\right) \\
\varphi_{G}\left(\Delta \varepsilon-p^{1}-y_{p}\right) \\
0
\end{array}\right)
\end{aligned}
$$

in the case of (4.4), and

$\boldsymbol{A}=\left(\begin{array}{c}0 \\ 0 \\ \theta-s-s^{\prime}\end{array}\right)+\frac{\theta(1+\cos \theta)}{2 \sin \theta}\left(\begin{array}{c}-d \sin E-y_{p} \\ d \cos E-x_{p} \\ 0\end{array}\right)+\frac{1}{2}\left(\begin{array}{c}\theta\left(d \cos E+x_{p}\right) \\ \theta\left(d \sin E-y_{p}\right) \\ 0\end{array}\right)$

in the case of (4.7). These three representations of the first order with respect to the precession/nutation and polar motion parameters may be useful to estimate different contributions (for instance, the relativistic ones) in the rigorous general transformations (65), (66) and (69). 


\section{Conclusion}

Relations (39)-(60) enable one to get the direct and inverse transformation between ICRS and GCRS for any of four pairs of currently employed time arguments (TCG, TCB, TCG and TT). In combination with relation (61) expressing geocentric position vectors in terms of barycentric quantities they may be used to take into account the relativistic third-body perturbations in the geocentric equations of motion of Earth's satellites and Earth's rotation equations. The direct and inverse transformation between GCRS and ITRS is performed now by means of Newtonian three-dimensional spatial rotations (63) under equivalent forms (65), (66) or (69). If one gets with the use of (61) the indirect relativistic perturbations in the Euler angles relating DGRSC (dynamically non-rotating geocentric RS) and ITRS then this transformation of the Newtonian form will become relativistic by its essence.

Acknowledgements. The participation of Dr. Jean-Louis Simon (IMCCE) in the final completing of this work and preparing it for publication is greatly acknowledged. The second author is thankful to the referee Dr. Nicole Capitaine for valuable comments that helped improve the manuscript (VAB).

\section{Appendix A: Rotation formula}

In this Appendix we derive straightforwardly the relations (68)-(71) used in Sect. 4. The mutual Newtonian three-dimensional rotation of the spatial axes of two reference systems

$\boldsymbol{y}=R \boldsymbol{x}$

is described by some orthogonal rotation matrix $R$. The inverse transformation is given by the transposed matrix $R^{\mathrm{T}}=R^{-1}$. The elements of the rotation matrix satisfy the relations

$R_{i k} R_{j k}=\delta_{i j}, \quad \varepsilon_{i j k} R_{j m} R_{k n}=\varepsilon_{l m n} R_{i l}$.

Any rotation matrix may be represented in terms of a skew-symmetric matrix $T$ as follows (Pars 1965):

$R=(E-T)^{-1}(E+T)=(E+T)(E-T)^{-1}$.

Matrix $T$ is composed of the components of the rotation vector $T^{i}$

$T^{i}=\frac{1}{2} \varepsilon_{i j k} T^{j k}, \quad T^{i j}=\varepsilon_{i j k} T^{k}$

(cf. matrix $F$ and vector $F^{i}$ of geodetic rotation (14). The inversion of (A.3) results in

$T=-(E-R)(E+R)^{-1}=-(E+R)^{-1}(E-R)$.

The structure (A.4) of matrix $T$ enables one to find easily the elements of the inverse matrix

$(E-T)_{i j}^{-1}=\frac{1}{1+|\boldsymbol{T}|^{2}}\left(\delta_{i j}+T^{i j}+T^{i} T^{j}\right)$.

Then from (A.3) one gets the representation of the elements of the rotation matrix in terms of the corresponding rotation vector

$R_{i j}=\frac{1}{1+|\boldsymbol{T}|^{2}}\left[\left(1-|\boldsymbol{T}|^{2}\right) \delta_{i j}+2 T^{i} T^{j}+2 \varepsilon_{i j k} T^{k}\right]$.

The rotation vector admits the representation (Pars 1965)

$\boldsymbol{T}=\boldsymbol{n} \tan \frac{\alpha}{2}$,

$\alpha$ and $\boldsymbol{n}$ being the rotation angle and unit rotation vector, respectively. Putting

$\boldsymbol{N}=\alpha \boldsymbol{n}$

one obtains from (A.7) the representation of the rotation matrix $R=R(N)$ as follows:

$R_{i j}(N)=\cos \alpha \delta_{i j}+(1-\cos \alpha) n^{i} n^{j}+\sin \alpha \varepsilon_{i j k} n^{k}$.

By multiplying both sides by $x^{j}$ one gets for any rotation vector $\boldsymbol{A}$

$R(\boldsymbol{A}) \boldsymbol{x}=\cos a \boldsymbol{x}-\sin a(\hat{\boldsymbol{A}} \times \boldsymbol{x})+(1-\cos a)(\hat{\boldsymbol{A}} \boldsymbol{x}) \hat{\boldsymbol{A}}$.

By applying here the formula for the double cross product one gets (70). For small vector $\boldsymbol{A}$ (small $a=|\boldsymbol{A}|$ ) there results within the second-order accuracy

$R(\boldsymbol{A}) \boldsymbol{x} \approx \boldsymbol{x}-(\boldsymbol{A} \times \boldsymbol{x})+\frac{1}{2}[\boldsymbol{A} \times(\boldsymbol{A} \times \boldsymbol{x})]$. 
The second small rotation $R(\boldsymbol{B})$ results in

$R(\boldsymbol{B}) R(\boldsymbol{A}) \boldsymbol{x} \approx R(\boldsymbol{A}) \boldsymbol{x}-(\boldsymbol{B} \times R(\boldsymbol{A}) \boldsymbol{x})+\frac{1}{2}[\boldsymbol{B} \times(\boldsymbol{B} \times R(\boldsymbol{A}) \boldsymbol{x})]$

or else

$R(\boldsymbol{B}) R(\boldsymbol{A}) \boldsymbol{x} \approx \boldsymbol{x}-(\boldsymbol{A} \times \boldsymbol{x})-(\boldsymbol{B} \times \boldsymbol{x})+\frac{1}{2}[\boldsymbol{A} \times(\boldsymbol{A} \times \boldsymbol{x})]+\frac{1}{2}[\boldsymbol{B} \times(\boldsymbol{B} \times \boldsymbol{x})]+[\boldsymbol{B} \times(\boldsymbol{A} \times \boldsymbol{x})]$.

After easy vector algebra manipulation the latter relation is transformed into

$R(\boldsymbol{B}) R(\boldsymbol{A}) \boldsymbol{x} \approx \boldsymbol{x}-(\boldsymbol{A} \times \boldsymbol{x})-(\boldsymbol{B} \times \boldsymbol{x})-\frac{1}{2}[(\boldsymbol{A} \times \boldsymbol{B}) \times \boldsymbol{x}]+\frac{1}{2}[(\boldsymbol{A}+\boldsymbol{B}) \times((\boldsymbol{A}+\boldsymbol{B}) \times \boldsymbol{x})]$

coinciding with the rotation formula

$R\left(\boldsymbol{A}+\boldsymbol{B}+\frac{1}{2}(\boldsymbol{A} \times \boldsymbol{B})\right) \boldsymbol{x} \approx \boldsymbol{x}-\left[\left(\boldsymbol{A}+\boldsymbol{B}+\frac{1}{2}(\boldsymbol{A} \times \boldsymbol{B})\right) \times \boldsymbol{x}\right]+\frac{1}{2}[(\boldsymbol{A}+\boldsymbol{B}) \times((\boldsymbol{A}+\boldsymbol{B}) \times \boldsymbol{x})]$.

Coincidence of the right-hand members of (A.15) and (A.16) proves (73).

Returning now to the general formula (A.10) and applying it for rotation about coordinate axes by putting $N=\alpha \boldsymbol{e} k(k=1,2,3)$ one gets

$R_{i j}\left(\alpha \boldsymbol{e}_{k}\right)=\cos \alpha \delta_{i j}+(1-\cos \alpha) \delta_{i k} \delta_{j k}+\sin \alpha \varepsilon_{i j k} \quad$ (no summation over $\left.k !\right)$.

Matrix elements (A.17) are just elements of the rotation matrices (17) proving (71).

It remains to prove (72). Considering that for any rotation matrix $M$, by virtue of (A.2)

$|M N|^{2}=M_{i j} N^{j} M_{i k} N^{k}=\delta_{j k} N^{j} N^{k}=|N|^{2}$

one gets from (A.10)

$R_{i j}(M N)=\cos \alpha \delta_{i j}+(1-\cos \alpha) M_{i r} n^{r} M_{j s} n^{s}+\sin \alpha \varepsilon_{i j r} M_{r s} n^{s}$.

Multiplying both sides by $M_{j k}$ and using (A.2) one obtains

$R_{i j}(M N) M_{j k}=\cos \alpha M_{i k}+(1-\cos \alpha) M_{i r} n^{r} n^{k}+\sin \alpha \varepsilon_{r k s} M_{i r} n^{s}$.

Using (A.10) it is easy to see that the right-hand member of (A.19) coincides with $M_{i j} R^{j k}(N)$. Hence,

$M R(N)=R(M N) M$.

This relation is equivalent to (72).

Let us derive now the composite rotation formulae (74) and (75). Combining the general rotation formula (70) for any rotation vector $\boldsymbol{A}$ with the first-order rotation formula for small vector $\boldsymbol{B}$

$R(\boldsymbol{B}) \boldsymbol{x}=\boldsymbol{x}+(\boldsymbol{x} \times \boldsymbol{B})$.

one gets for the sequences of rotations:

$$
\begin{aligned}
R(\boldsymbol{B}) R(\boldsymbol{A}) \boldsymbol{x}= & R(\boldsymbol{A}) \boldsymbol{x}+[R(\boldsymbol{A}) \boldsymbol{x} \times \boldsymbol{B}]=R(\boldsymbol{A}) \boldsymbol{x}+(\hat{\boldsymbol{A}} \boldsymbol{x})(\hat{\boldsymbol{A}} \times \boldsymbol{B}) \\
& +\sin a[(\boldsymbol{B} \boldsymbol{x}) \hat{\boldsymbol{A}}-(\hat{\boldsymbol{A}} \boldsymbol{B}) \boldsymbol{x}]+\cos a[(\boldsymbol{x} \times \boldsymbol{B})+(\hat{\boldsymbol{A}} \boldsymbol{x})(\boldsymbol{B} \times \hat{\boldsymbol{A}})] \\
R(\boldsymbol{A}) R(\boldsymbol{B}) \boldsymbol{x}= & (\hat{\boldsymbol{A}} R(\boldsymbol{B}) \boldsymbol{x}) \hat{\boldsymbol{A}}-\sin a[\hat{\boldsymbol{A}} \times R(\boldsymbol{B}) \boldsymbol{x}]+\cos a[R(\boldsymbol{B}) \boldsymbol{x}-\hat{\boldsymbol{A}}(\hat{\boldsymbol{A}} R(\boldsymbol{B}) \boldsymbol{x})]=R(\boldsymbol{A}) \boldsymbol{x}+(\boldsymbol{x} \times \boldsymbol{B}) \\
& +(\hat{\boldsymbol{A}} \boldsymbol{x})(\boldsymbol{B} \times \hat{\boldsymbol{A}})+(\hat{\boldsymbol{A}} \boldsymbol{B})(\hat{\boldsymbol{A}} \times \boldsymbol{x})+\sin a[(\hat{\boldsymbol{A}} \boldsymbol{x}) \boldsymbol{B}-(\hat{\boldsymbol{A}} \boldsymbol{B}) \boldsymbol{x}]+\cos a[(\hat{\boldsymbol{A}} \boldsymbol{x})(\hat{\boldsymbol{A}} \times \boldsymbol{B})-(\hat{\boldsymbol{A}} \boldsymbol{B})(\hat{\boldsymbol{A}} \times \boldsymbol{x})] .
\end{aligned}
$$

If vector $\boldsymbol{A}$ has variation $\delta \boldsymbol{A}$ then the variation of the rotation formula will be

$R(\boldsymbol{A}+\delta \boldsymbol{A}) \boldsymbol{x}=R(\boldsymbol{A}) \boldsymbol{x}+\Delta(\delta \boldsymbol{A})$.

Considering that

$\delta a=\hat{A} \delta \boldsymbol{A}, \quad \delta \hat{\boldsymbol{A}}=\frac{1}{a}[\delta \boldsymbol{A}-(\hat{\boldsymbol{A}} \delta \boldsymbol{A}) \hat{\boldsymbol{A}}]$

one gets from (70)

$$
\begin{aligned}
\Delta(\delta \boldsymbol{A})= & \frac{1}{a}[(\hat{\boldsymbol{A}} \boldsymbol{x}) \delta \boldsymbol{A}-2(\hat{\boldsymbol{A}} \boldsymbol{x})(\hat{\boldsymbol{A}} \delta \boldsymbol{A}) \hat{\boldsymbol{A}}+(\boldsymbol{x} \delta \boldsymbol{A}) \hat{\boldsymbol{A}}]+\sin a\left[(\hat{\boldsymbol{A}} \boldsymbol{x})(\hat{\boldsymbol{A}} \delta \boldsymbol{A}) \hat{\boldsymbol{A}}-\boldsymbol{x}(\hat{\boldsymbol{A}} \delta \boldsymbol{A})+\frac{1}{a}(\boldsymbol{x} \times \delta \boldsymbol{A})\right. \\
& \left.+\frac{1}{a}(\hat{\boldsymbol{A}} \delta \boldsymbol{A})(\hat{\boldsymbol{A}} \times \boldsymbol{x})\right]+\frac{\cos a}{a}[2(\hat{\boldsymbol{A}} \boldsymbol{x})(\hat{\boldsymbol{A}} \delta \boldsymbol{A}) \hat{\boldsymbol{A}}-(\hat{\boldsymbol{A}} \boldsymbol{x}) \delta \boldsymbol{A}-(\boldsymbol{x} \delta \boldsymbol{A}) \hat{\boldsymbol{A}}-a(\hat{\boldsymbol{A}} \delta \boldsymbol{A})(\hat{\boldsymbol{A}} \times \boldsymbol{x})]
\end{aligned}
$$


This formula enables one to find three elementary variations as follows:

$$
\begin{aligned}
& \Delta(\boldsymbol{B})=\frac{1}{a}[(\hat{\boldsymbol{A}} \boldsymbol{x}) \boldsymbol{B}-2(\hat{\boldsymbol{A}} \boldsymbol{x})(\hat{\boldsymbol{A}} \boldsymbol{B}) \hat{\boldsymbol{A}}+(\boldsymbol{x} \boldsymbol{B}) \hat{\boldsymbol{A}}]+\sin a\left[(\hat{\boldsymbol{A}} \boldsymbol{x})(\hat{\boldsymbol{A}} \boldsymbol{B}) \hat{\boldsymbol{A}}-\boldsymbol{x}(\hat{\boldsymbol{A}} \boldsymbol{B})+\frac{1}{a}(\boldsymbol{x} \times B)+\frac{1}{a}(\hat{\boldsymbol{A}} \boldsymbol{B})(\hat{\boldsymbol{A}} \times \boldsymbol{x})\right] \\
& +\frac{\cos a}{a}[2(\hat{A} \boldsymbol{x})(\hat{\boldsymbol{A}} \boldsymbol{B}) \hat{\boldsymbol{A}}-(\hat{\boldsymbol{A}} \boldsymbol{x}) \boldsymbol{B}-(\boldsymbol{x} \boldsymbol{B}) \hat{\boldsymbol{A}}-a(\hat{\boldsymbol{A}} \boldsymbol{B})(\hat{\boldsymbol{A}} \times \boldsymbol{x})], \\
& \Delta(\hat{A}(\hat{A} \boldsymbol{B}))=\sin a(\hat{\boldsymbol{A}} \boldsymbol{B})[(\hat{\boldsymbol{A}} \boldsymbol{x}) \hat{\boldsymbol{A}}-\boldsymbol{x}]+\cos a(\hat{\boldsymbol{A}} \boldsymbol{B})(\boldsymbol{x} \times \hat{\boldsymbol{A}}],
\end{aligned}
$$

and

$\Delta(\hat{\boldsymbol{A}} \times \boldsymbol{B})=\frac{1-\cos a}{a}[(\boldsymbol{B} \times \boldsymbol{x})+2(\hat{\boldsymbol{A}} \boldsymbol{x})(\hat{\boldsymbol{A}} \times \boldsymbol{B})+(\hat{\boldsymbol{A}} \boldsymbol{B})(\boldsymbol{x} \times \hat{\boldsymbol{A}})]+\frac{\sin a}{a}[(\boldsymbol{B} \boldsymbol{x}) \hat{\boldsymbol{A}}-(\hat{\boldsymbol{A}} \boldsymbol{x}) \boldsymbol{B}]$.

From these elementary variations it is easy to see that

$R(\boldsymbol{B}) R(\boldsymbol{A}) \boldsymbol{x}-R(\boldsymbol{A}) R(\boldsymbol{B}) \boldsymbol{x}=\Delta(\boldsymbol{A} \times \boldsymbol{B})$,

and

$\Delta(\boldsymbol{B})-\Delta(\hat{A}(\hat{A} \boldsymbol{B}))=\frac{1-\cos a}{a}[(\hat{\boldsymbol{A}} \boldsymbol{x}) \boldsymbol{B}-2(\hat{A} \boldsymbol{x})(\hat{A} \boldsymbol{B}) \hat{\boldsymbol{A}}+(\boldsymbol{x} \boldsymbol{B}) \hat{A}]+\frac{\sin a}{a}[(\boldsymbol{x} \times \boldsymbol{B})+(\hat{A} \boldsymbol{B})(\hat{A} \times \boldsymbol{x})]$.

From (A.28) and (A.30) there results

$\Delta(\boldsymbol{A} \times \boldsymbol{B})+\frac{a(1+\cos a)}{\sin a}[\Delta(\boldsymbol{B})-\Delta(\hat{A}(\hat{A} \boldsymbol{B}))]=2[R(\boldsymbol{B}) R(\boldsymbol{A}) \boldsymbol{x}-R(\boldsymbol{A}) \boldsymbol{x}-\Delta(\hat{\boldsymbol{A}}(\hat{\boldsymbol{A}} \boldsymbol{B}))]$.

Equations (A.31) and (A.29) result in the final composite first-order (with respect to B) formulae (74) and (75).

\section{Appendix B: VSOP data for the transformation between BCRS and GCRS}

This Appendix contains the initial terms of the series for $\boldsymbol{x}_{E}, \boldsymbol{v}_{E}, c^{-2} \boldsymbol{a}_{E}, c^{-2} \bar{U}_{E}\left(t, \boldsymbol{x}_{E}\right), c^{-2} A_{p}, c^{-2} A_{p} \boldsymbol{v}_{E}$ and $c^{-2} \boldsymbol{F}$ in function of $t=$ TDB as computed with VSOP theories. These series are to be used to evaluate explicitly the coefficients in (56)-(60). For the sake of completeness we give also the series for $c^{-2} \dot{\boldsymbol{F}}$ needed when considering the equations of motion in GCRS. All values are given using the astronomical unit as the unit of length and 1000 Julian years (365250 Julian days) as the unit of time. The value of $L_{C}$ obtained with the VSOP solution reads

$L_{C}=1.480826855667 \times 10^{-8}$.

In integrating the equations for $\dot{A}$ and $\dot{\boldsymbol{F}}$ the additive constants were introduced to annul these quantities for the 1977 Origin and $\mathbf{J} 2000.0$, respectively.

All series are presented in form of (31), i.e.

$x_{E}^{i}(t)=\sum_{\alpha} t^{\alpha}\left[\sum_{k} X_{i k}^{\alpha} \cos \left(\psi_{k}^{\alpha}+v_{k}^{\alpha} t\right)\right]$.

The data in the 6-column Tables 1-20 read: ordinal number of the term, components of the trigonometric argument (mean longitudes of eight major planets from Mercury to Neptune and arguments $D, F, l$ of the lunar theory) given to show the physical meaning of the term, coefficient $X$, the phase angle $\psi$ of the argument, the frequency $v$ of the argument and exposant $\alpha$ of power of $t$.

The truncation level is as follows:

0.5 E-03 au over 1000 yrs for $\boldsymbol{x}_{E}$ (Tables $\left.1-3\right)$,

$0.5 \mathrm{au} / 1000$ yrs over 1000 yrs for $\boldsymbol{v}_{E}$ (Tables 4-6),

0.4 E-12 over 1000 yrs for $c^{-2} \boldsymbol{a}_{E}$ (Tables 7-9),

$0.15 \mathrm{E}-12$ over $1000 \mathrm{yrs}$ for $c^{-2} \bar{U}_{E}\left(t, \boldsymbol{x}_{E}\right)$ (Table 10 ),

$0.5 \mathrm{E}-16$ over $1000 \mathrm{yrs}$ for $c^{-2} A_{p}$ (Table 11 ),

$0.8 \mathrm{E}-12$ over $1000 \mathrm{yrs}$ for $c^{-2} A_{p} \boldsymbol{v}_{E}$ (Tables 12-14),

$0.1 \mathrm{E}-12$ over 1000 yrs for $c^{-2} \boldsymbol{F}$ (Tables $15-17$ ),

0.1 E-8 over 1000 yrs for $c^{-2} \dot{\boldsymbol{F}}$ (Tables 18-20). 


\section{References}

Bretagnon, P. 2002, Trans. Inst. Appl. Astron., 8, 33

Bretagnon, P., \& Francou, G. 1988, A\&A, 202, 309

Bretagnon, P., Rocher, P., \& Simon, J. L. 1997, A\&A, 319, 305

Bretagnon, P., Francou, G., Rocher, P., \& Simon, J. L. 1998, A\&A, 329, 329

Bretagnon, P., Fienga, A., \& Simon, J.-L. 2003, A\&A, 400, 785

Brumberg, V. A. 1991, Essential Relativistic Celestial Mechanics (Bristol: Hilger)

Brumberg, V. A. 1996, in Earth Rotation, Reference Systems in Geodynamics and Solar System (Journées 1995), ed. N. Capitaine, B. Kolaczek, \& S. Debarbat, Warsaw, 29

Brumberg, V. A. 1997, in Dynamics and Astrometry of Natural and Artificial Celestial Bodies, ed. I. M. Wytrzyszczak, J. H. Lieske, \& R. A. Feldman, IAU Coll. 165 (Poznan, 1996, Kluwer), 439

Brumberg, V. A., \& Bretagnon, P. 2001, Trans. Inst. Appl. Astron. 6, 170 (in Russian)

Brumberg, V. A., \& Groten, E. 2001a, A\&A, 367, 1070

Brumberg, V. A., \& Groten, E. 2001b, J. Geodesy, 75, 673

Brumberg, V. A., Bretagnon, P., \& Guinot, B. 1996, Celes. Mech. Dyn. Astron., 64, 231

BDL 1997, Introduction aux éphémérides astronomiques, Supplément explicatif à la Connaissance des Temps, ed. J.-L. Simon, M. Chapront-Touzé, B. Morando, \& W. Thuillot (Bureau des Longitudes)

Capitaine, N., Guinot, B., \& McCarthy, D. D. 2000, A\&A, 355, 398

Fairhead, L., \& Bretagnon, P. 1990, A\&A, 229, 240

Folkner, W. M., Charlot, P., Finger, M. H., et al. 1994, A\&A, 287, 279

Fukushima, T. 1995, A\&A, 294, 895

Guinot, B. 2000, in J2000, A fundamental epoch for origins of reference systems and astronomical models (Journées 2000), Obs. de Paris, ed. N. Capitaine, 209

IAU 2001, IAU Information Bull., 88, 28 (Errata: ibid., 89, 4, 2001)

IERS: 2002, Proc. of the IERS Workshop on the Implementation of the New IAU Resolutions, IERS Techn. Note No. 29, ed. N. Capitaine, D. Gambis, D. D. McCarthy, et al.

IERS 2003, IERS Conventions 2000, http://maia.usno.navy.mil/conv2000.html, in press

Irwin, A. W., \& Fukushima, T. 1999, A\&AS, 348, 642

Moritz, H., \& Mueller, I. I. 1987, Earth Rotation (New York: Ungar)

Pars, L. A. 1965, Analytical Dynamics, Chap. 7 (London: Heinemann)

Soffel, M., Klioner, S. A., Petit, G., et al. 2003, AJ, in press

Vokrouhlický, D. 1995, Phys. Rev. D, 52, 6894 